\title{
Almost contraction mappings in cone $b$-metric spaces over Banach algebras
}

\author{
Faruk Develi* (D), Muttalip Özavşar (D) \\ Department of Mathematics, Yildiz Technical University, 34210 Istanbul, Turkey
}

\begin{abstract}
We introduce the condition of being Cauchy for a sequence in cone $b$-metric spaces (cbms) over Banach algebras. Based on this result, we extend almost mappings in cone metric spaces over Banach algebras to cbms over Banach algebras and prove the related fixed point theorem. In addition, we apply our results to some applications to illustrate their usability.
\end{abstract}

Mathematics Subject Classification (2010). 47H10, 54H25

Keywords. cone $b$-metric spaces over Banach algebras, spectral radius, common fixed point

\section{Introduction}

In 1922, Banach proved a fixed point theorem known as Banach contraction principle, which is one of the most important tools in nonlinear analysis. It is well known that Banach contraction principle plays a vital role for existence and uniqueness theorems in different branches of analysis. For this reason, many people have been studied his result for distinct contractive type mappings. As an example, Jungck [7] initiated a study of common fixed points for a pair of self-mappings in 1976. Moreover, in 2004, Berinde [2] introduced almost contraction mappings, which contain the classes of many mappings such as Banach, Kannan, Chatterjea, and proved a related fixed point theorem.

On the other hand, the fixed point theory has been studied in distinct type metric spaces. For example, in 2007, Huang and Zhang [4] obtained some fixed point theorems in cone metric spaces over Banach spaces, where the distance of two elements is defined as a vector in an ordered Banach space. But, in 2010, Du [3] drew attention of authors to the equivalence between fixed point theorems in cone metric spaces and the usual metric spaces. Howeover, by taking the constants of contractive type mappings as a vector instead of a real number, in 2013, Liu and $\mathrm{Xu}$ [10] presented fixed point theorems of these mappings in cone metric spaces over Banach algebras by using tools of spectral radius and normal solid cones, and also they gave an example to confirm that it is not equivalent to versions in the usual metric spaces. Afterwards, in 2014, Xu and Radenovic [16] obtained the results given in [10] by removing the normality condition of cone. Then, in 2015, the notion of cone $b$-metric space over Banach algebras was introduced by Huang and Radenovic [5]

\footnotetext{
* Corresponding Author.

Email addresses: fdeveli@yildiz.edu.tr (F. Develi), mozavsar@yildiz.edu.tr (M. Özavşar)

Received: 23.11.2018; Accepted: 10.03.2020
} 
as a generalization of cone metric spaces over Banach algebras. In addition to these, In 2017, Suzuki [15] gave a very useful condition for the Cauchyness on sequences in $b$-metric spaces.

Based on the results given in [16] and [2], in 2018, Özavşar [11] studied almost contraction mappings in the setting of cone metric spaces over Banach algebras. In this work, inspired by works [15] and [11], we firstly present the condition of being Cauchy for a sequence in cone $b$-metric spaces over Banach algebras. Next we prove common fixed point theorems for two mappings satisfying an almost contractive condition in cone $b$-metric spaces over Banach algebras. Finally, as applications of our main results, we show the existence and uniqueness of solution for some systems of equations.

\section{Preliminaries}

A Banach algebra $A$ is a Banach space over $F=\{\mathbb{R}, \mathbb{C}\}$ together with an associative and distributive multiplication such that $\alpha(a b)=(\alpha a) b=a(\alpha b)$ and $\|a b\| \leq\|a\|\|b\|$ for all $a, b \in A, \alpha \in F$ where $\|$.$\| is the norm on A$. Let $A$ has a unit (i.e. multiplicative identity) $e$ such that $e a=a e=a$ for all $a \in A$. An element $a \in A$ is said to be invertible if there is an inverse element $b \in A$ such that $a b=b a=e$. The inverse of $a$ is denoted by $a^{-1}$. Now we shortly recall the spectral radius which is neccessary for main result. Let $A$ be a Banach algebra with a unit $e$. The spectral radius of $a \in A$ as follows:

$$
\rho(a)=\lim _{n \rightarrow \infty}\left\|a^{n}\right\|^{\frac{1}{n}} .
$$

If $\rho(a)<1$, then $e-a$ is invertible and the inverse of $e-a$ is given by

$$
(e-a)^{-1}=\sum_{i=0}^{\infty} a^{i}
$$

For more details, we refer the reader to Rudin (see [14]). Let $A$ be a Banach algebra with a unit $e$, and $\theta$ the zero element of $A$. Now let us recall the concepts of cone to obtain a partial ordered relation on $A$. A nonempty closed subset $P$ of $A$ is called a cone of $A$ if $\{\theta, e\} \subset P, P^{2}=P P \subset P, P \cap(-P)=\{\theta\}$ and $\alpha P+\beta P \subset P$ for all $\alpha, \beta \geq 0$. For a given cone $P \subset A$, we can define a partial ordering $\preceq$ as follow:

$$
a \preceq b \text { if and only if } b-a \in P .
$$

Furthermore, we will indicate that

$$
\begin{aligned}
& a \prec \quad b \text { iff } a \preceq b \text { and } a \neq b, \\
& a \ll b \text { iff } b-a \in \operatorname{int} P,
\end{aligned}
$$

where int $P$ denotes the interior of $P$. If $i n t P \neq \emptyset$ then $P$ is called a solid cone (see [4]). From now on, we always suppose that $A$ is a Banach algebra with unit $e, P$ is a solid cone in $A$, and $\preceq$ is a partial ordering with respect to $P$.

Lemma $2.1([14])$. Let $a, b \in A$ with $a b=b a$. Then the following conditions satisfy:

(i) $\rho(a b) \leq \rho(a) \rho(b)$,

(ii) $\rho(a+b) \leq \rho(a)+\rho(b)$.

Definition $2.2([5])$. Let $X$ be a nonempty set and $1 \leq s$ be a constant. Let $d$ be a mapping from $X \times X$ into $A$. Then $(X, d)$ is said to be a cone $b$-metric space over $A$ if the following hold

cbm1: $\theta \preceq d(x, y)$ and $d(x, y)=\theta$ if and only if $x=y$

cbm2: $d(x, y)=d(y, x)$

cbm3: $d(x, y) \preceq s(d(x, z)+d(z, x))$

for all $x, y, z \in X$. 
If we take $s=1$, every cone $b$-metric space over $A$ is obviously a cone metric space over $A$.

Definition $2.3([5])$. Let $(X, d)$ be a cone $b$-metric space over $A, x \in X$ and $\left\{x_{n}\right\}$ be sequence in $X$. Then:

(1) $\left\{x_{n}\right\}$ convergens to $x$ whenever for each $c \in A$ with $\theta \ll c$ there is a naturel number $N$ such that $d\left(x_{n}, x\right) \ll c$ for all $n \geq N$. This is denoted by $\lim _{n \rightarrow \infty} x_{n}=x$ or $x_{n} \rightarrow x, n \rightarrow \infty$.

(2) $\left\{x_{n}\right\}$ is a Cauchy sequence whenever for each $c \in A$ with $\theta \ll c$ there is a naturel number $N$ such that $d\left(x_{n}, x_{m}\right) \ll c$ for all $n, m \geq N$.

(3) $(X, d)$ said to be complete if every Cauchy sequence $\left\{x_{n}\right\}$ in $X$ is convergent.

Definition $2.4([1,8])$. Let $S, T: X \rightarrow X$ be mappings on $X$.

(1) If $y=S x=T x$ for some $x \in X$, then $x$ is said to be a coincidence point of $S$ and $T$, and $y$ is said a point of coincidence of $S$ and $T$.

(2) The pair $\{S, T\}$ is said weakly compatible if $S$ and $T$ commute at all of coincidence points, that is, $S T x=T S x$ for all $x \in\{x \in X: S x=T x\}$.

Proposition 2.5 ([1]). Let $S$ and $T$ be weakly compatible selfmaps of a set $X$. If $S$ and $T$ have a unique point of coincidence $y=S x=T x$, then $y$ is the unique common fixed point of $S$ and $T$.

Definition $2.6([16])$. A sequence $\left\{u_{n}\right\} \subset P$ is a $c$-sequence if for each $\theta \ll c$ there exists $n_{0} \in \mathbb{N}$ such that $u_{n} \ll c$ for $n>n_{0}$.

Let $(X, d)$ complete cone $b$-metric space over $A$. We obtain from the above definition that if $\left\{x_{n}\right\}$ convergens to $x \in X,\left\{d\left(x_{n}, x\right)\right\}$ is a $c$-sequence.

Lemma 2.7 ([5]). If $\rho(a)<1$, then $\left\{a^{n}\right\}$ is a c-sequence.

Lemma 2.8 ([16]). If a sequence $\left\{u_{n}\right\}$ in $P$ is a c-sequence, then $\left\{k u_{n}\right\}$ is a c-sequence where $k \in P$.

Lemma $2.9([9])$. If two sequences $\left\{u_{n}\right\}$ and $\left\{v_{n}\right\}$ in $P$ are $c$-sequences, then $\left\{\alpha u_{n}+\beta v_{n}\right\}$ is a c-sequence where $\alpha, \beta>0$.

Lemma 2.10 ([13]). The following conditions hold:

(i) If $a \preceq b$ and $b \ll c$, then $a \ll c$.

(ii) If $\theta \preceq a \ll c$ for each $\theta \ll c$, then $a=\theta$.

Lemma $2.11([15])$. Let $(X, d)$ be a cone b-metric space and $f(n)=-\left[-\log _{2} n\right]$ which is a function from $\mathbb{N}$ to $\mathbb{N} \cup\{0\}$. Then, the following condition holds

$$
d\left(z_{0}, z_{n}\right) \preceq s^{f(n)} \sum_{i=0}^{n-1} d\left(z_{i}, z_{i+1}\right)
$$

for all $\left(z_{0}, \ldots, z_{n}\right) \in X^{n+1}$.

Proof. The proof is seen clearly by following similar steps in [15].

\section{Main results}

In this section, we establish a common fixed point result for the following contraction mapping with vector constant:

$$
d(T x, T y) \preceq k d(S x, S y)+l d(S y, T x)
$$

for all $x, y \in X$ where $k \in P$ with $\rho(k)<1$ and $\theta \preceq l$. This contraction is the version of almost contraction mapping for a pair of self-maps, which is important in terms of containing some well known contractive type mappings (see [2] and [11]), in cone metric 
space over Banach algebra. Before giving the related result in cone $b$-metric space over Banach algebra, we need the following significant lemma.

Lemma 3.1. Let $(X, d)$ be a complete cone b-metric space over A. Assume that $\left\{z_{n}\right\}$ is a sequence in $X$ and there exists $h \in A$ with $\rho(h)<1$ such that for $n=1,2, \ldots$, the following inequality holds:

$$
d\left(z_{n}, z_{n+1}\right) \preceq h d\left(z_{n-1}, z_{n}\right) .
$$

Then, $\left\{z_{n}\right\}$ is a Cauchy sequence in $X$.

Proof. It follows from (3.1) that

$$
d\left(z_{n}, z_{n+1}\right) \preceq h d\left(z_{n-1}, z_{n}\right) \preceq \cdots \preceq h^{n} d\left(z_{0}, z_{1}\right) .
$$

By Lemma 2.1, it is clear that there is $\ell \in \mathbb{N}$ such that $\rho\left(s h^{2^{\ell}}\right)<1$. To show that $\left\{z_{n}\right\}$ is a Cauchy sequence, we will benefit from the function $f$ defined in Lemma 2.11. Let $\theta \ll c$ be given. Firstly, in the case of $n<m \leq n+2^{\ell}$, we obtain the following inequality from Lemma 2.11

$$
d\left(z_{n}, z_{m}\right) \preceq s^{f(m-n)} \sum_{i=n}^{m-1} d\left(z_{i}, z_{i+1}\right) .
$$

Since $f$ is nondecreasing and so $f(m-n) \leq f\left(2^{\ell}\right)=\ell$, by 4.2 , we get

$$
\begin{aligned}
d\left(z_{n}, z_{m}\right) & \preceq s^{\ell} \sum_{i=n}^{m-1} h^{i} d\left(z_{0}, z_{1}\right) \\
& =s^{\ell} h^{n}\left(\sum_{i=0}^{m-n-1} h^{i}\right) d\left(z_{0}, z_{1}\right) \\
& \preceq s^{\ell} h^{n}\left(\sum_{i=0}^{\infty} h^{i}\right) d\left(z_{0}, z_{1}\right)=s^{\ell} h^{n} C
\end{aligned}
$$

where $C=(e-h)^{-1} d\left(z_{0}, z_{1}\right)$. Setting $\alpha_{n}=s^{\ell} h^{n} C$, it is seen that $\alpha_{n}$ is a $c$-sequence according to Lemma 2.7, Lemma 2.8 and Lemma 2.9. Therefore, we have $N_{0} \in \mathbb{N}$ such that

$$
d\left(z_{n}, z_{m}\right) \preceq \alpha_{n} \ll c \text { for } n>N_{0} .
$$

Secondly, in the case of $n+2^{\ell}<m$, we use (cbm3) by setting $\gamma=\left[(m-n) / 2^{\ell}\right]$ as follows:

$$
d\left(z_{n}, z_{m}\right) \preceq \sum_{i=0}^{\gamma-1} s^{i+1} d\left(z_{n+i 2^{\ell}}, z_{n+(i+1) 2^{\ell}}\right)+s^{\gamma} d\left(z_{n+\gamma 2^{\ell}}, z_{m}\right) .
$$

Note that $n+(i+1) 2^{\ell}-n-i 2^{\ell} \leq 2^{\ell}$ and $m-n-\gamma 2^{\ell} \leq 2^{\ell}$. Thus, the following inequalities are obtained from (3.3)

$$
\begin{array}{r}
d\left(z_{n+i 2^{\ell}}, z_{n+(i+1) 2^{\ell}}\right) \preceq s^{\ell} h^{n+i 2^{\ell}} C, \\
d\left(z_{n+\gamma 2^{\ell}}, z_{m}\right) \preceq s^{\ell} h^{n+\gamma 2^{\ell}} C .
\end{array}
$$

By substituting the inequalities in (3.6) to (3.5), we get

$$
\begin{aligned}
d\left(z_{n}, z_{m}\right) & \preceq \sum_{i=0}^{\gamma-1} s^{i+1+\ell} h^{n+i 2^{\ell}} C+s^{\gamma+\ell} h^{n+\gamma 2^{\ell}} C \\
& \preceq s^{\ell+1}\left(\sum_{i=0}^{\gamma} s^{i} h^{n+i 2^{\ell}}\right) C=s^{\ell+1}\left(\sum_{i=0}^{\gamma}\left(s h^{2^{\ell}}\right)^{i}\right) h^{n} C \\
& \preceq s^{\ell+1}\left(\sum_{i=0}^{\infty}\left(s h^{2^{\ell}}\right)^{i}\right) h^{n} C .
\end{aligned}
$$


Thus, since $\rho\left(s h^{2^{\ell}}\right)<1$,

$$
d\left(z_{n}, z_{m}\right) \preceq s^{\ell+1}\left(e-s h^{2^{\ell}}\right)^{-1} h^{n} C .
$$

Letting $\beta_{n}=s^{\ell+1}\left(e-s h^{2^{\ell}}\right)^{-1} h^{n} C$, it is obvious that $\beta_{n}$ is a $c$-sequence according to Lemma 2.7, Lemma 2.8 and Lemma 2.9. So we have $N_{1} \in \mathbb{N}$ such that

$$
d\left(z_{n}, z_{m}\right) \preceq \beta_{n} \ll c \text { for } n>N_{1} .
$$

Finally, taking $N=\max \left\{N_{0}, N_{1}\right\}$, we obtain from (3.4) and (3.7) that the following condition

$$
d\left(z_{n}, z_{m}\right) \ll c
$$

holds for all $m, n$ with $n<m$. Thus, $\left\{z_{n}\right\}$ is a Cauchy sequence.

Remark 3.2. Comparing Lemma 2.2 in [6] and Lemma 3.1, you can easily see that we replace the condition $\rho(k)<\frac{1}{s}$ with $\rho(k)<1$.

Corollary 3.3 ([12]). Let $(X, d)$ be a complete cone metric space over A. Assume that $\left\{z_{n}\right\}$ is a sequence in $X$ and there exists $h \in A$ with $\rho(h)<1$ such that for $n=1,2, \ldots$, the following inequality holds:

$$
d\left(z_{n}, z_{n+1}\right) \preceq h d\left(z_{n-1}, z_{n}\right) .
$$

Then, $\left\{z_{n}\right\}$ is a Cauchy sequence in $X$.

Theorem 3.4. Let $(X, d)$ be a cone b-metric space over A. Assume that two mappings $S, T: X \rightarrow X$ satisfy $T X \subset S X$ and $S X$ is a complete subspace of $X$. If the following condition holds:

$$
d(T x, T y) \preceq k d(S x, S y)+l d(S y, T x)
$$

for all $x, y \in X$ where $k \in P$ with $\rho(k)<1$ and $\theta \preceq l$, then $\{S, T\}$ has at least one point of coincidence in $X$.

Proof. Let $x_{0}$ be an arbitrary point in $X$. Since $T X \subset S X$, there exists $x_{1} \in X$ such that $T x_{0}=S x_{1}$. By continuing this process, we get the sequence $\left\{x_{n}\right\}$ in the following way

$$
T x_{n}=S x_{n+1} \text { for } n=0,1,2, \ldots
$$

By setting $x=x_{n-1}$ and $y=x_{n}$ in (3.9), we have

$$
d\left(S x_{n}, S x_{n+1}\right)=d\left(T x_{n-1}, T x_{n}\right) \preceq k d\left(S x_{n-1}, S x_{n}\right)+l d\left(S x_{n}, T x_{n-1}\right),
$$

which implies that

$$
d\left(S x_{n}, S x_{n+1}\right) \preceq k d\left(S x_{n-1}, S x_{n}\right) .
$$

Thus, considering Lemma 3.1, we easily say that $\left\{S x_{n}\right\}$ is a Cauchy sequence. Since $S X$ is a complete subspace of $X$, there exists $q \in S X$ such that $S x_{n} \rightarrow q=S p$ for some $p \in X$ and also $T x_{n} \rightarrow q$. Let us show $T p=q$. From (3.9), we obtain

$$
\begin{aligned}
d\left(S x_{n+1}, T p\right) & =d\left(T x_{n}, T p\right) \preceq k d\left(S x_{n}, S p\right)+l d\left(S p, T x_{n}\right) \\
& =k d\left(S x_{n}, q\right)+l d\left(q, T x_{n}\right) .
\end{aligned}
$$

Since $\left\{d\left(S x_{n}, q\right)\right\}$ and $\left\{d\left(T x_{n}, q\right)\right\}$ are $c$-sequences, we get that $\left\{d\left(S x_{n+1}, T p\right)\right\}$ is a $c$ sequence according to Lemma 2.8 and Lemma 2.9. Therefore, $S x_{n}$ converges to $T p$ and then $S p=T p=q$.

Theorem 3.5. Let the conditions in Theorem 3.4 be supplied. In addition to this, suppose that there exist $\alpha \in P$ with $\rho(\alpha)<1$ and some $\theta \preceq L$ such that for all $x, y \in X$

$$
d(T x, T y) \preceq \alpha d(S x, S y)+L d(S x, T x),
$$

then $\{S, T\}$ has a unique point of coincidence in $X$. Moreover, if $\{S, T\}$ is weakly compatible pair, then $S$ and $T$ have a unique common fixed point. 
Proof. We know from Theorem 3.4 that $S$ and $T$ have a point of coincidence $q$ in $X$, that is, $q=S p=T p$. We shall prove that $S$ and $T$ have a unique point of coincidence. To show its uniqueness, we first suppose that there is another $q^{\prime}$ such that $q^{\prime}=S p^{\prime}=T p^{\prime}$ for some $p^{\prime} \in X$. Then, we get by (3.10)

$$
d\left(S p, S p^{\prime}\right)=d\left(T p, T p^{\prime}\right) \preceq \alpha\left(S p, S p^{\prime}\right),
$$

which implies that

$$
d\left(q, q^{\prime}\right) \preceq \alpha d\left(q, q^{\prime}\right) \preceq \cdots \preceq \alpha^{n} d\left(q, q^{\prime}\right) .
$$

By using Lemma 2.7 and Lemma $2.8, \alpha^{n} d\left(q, q^{\prime}\right)$ is a $c$-sequence and by Lemma 2.10 , it is easy to see that $d\left(q, q^{\prime}\right)=\theta$, that is, $q=q^{\prime}$. This means that $\{S, T\}$ has a unique point of coincidence. Finally, if $\{S, T\}$ is weakly compatible, it follows from Proposition 2.5 that $q$ is a unique common fixed point of these two mappings.

Corollary 3.6. Let $(X, d)$ be a cone b-metric space over A. Assume that two mappings $S, T: X \rightarrow X$ satisfy $T X \subset S X$ and $S X$ is a complete subspace of $X$. If there is $k \in P$ with $\rho(k)<1$ such that for all $x, y \in X$

$$
d(T x, T y) \preceq k d(S x, S y),
$$

then $S$ and $T$ have a unique point of coincidence in $X$. Moreover, if $\{S, T\}$ is weakly compatible pair, then $S$ and $T$ have a unique common fixed point.

\section{Conclusion and applications}

In this paper, we extend almost mappings and the related fixed point theorems given in [11] to cone $b$-metric spaces over Banach algebras. Furthermore, we notice that Corollary 3.6 given above presents a sharp generalization of Corallary 2.10 in [5] by replacing the condition $\rho(k)<\frac{1}{s}$ with $\rho(k)<1$. Now, by using the obtained results, we first show the existence and uniqueness of solution for the following system of integral equations with a weaker condition than one given in [5]:

$$
\begin{aligned}
& x(t)=\int_{a}^{t} f(s, x(s)) d s \\
& x(t)=\int_{a}^{t} x(s) d s,
\end{aligned}
$$

where $t \in[a, b]$ and $f:[a, b] \times \mathbb{R} \rightarrow \mathbb{R}$ is a continuous function.

Theorem 4.1. Let $L_{p}[a, b]=\left\{x=x(t): \int_{a}^{b}|x(t)|^{p} d t<\infty\right\}(0<p<1)$. Suppose that $X=L_{p}[a, b]$ and $T, S: X \rightarrow X$ are mappings defined as follows:

$$
\begin{aligned}
T x(t) & =\int_{a}^{t} f(s, x(s)) d s \\
S x(t) & =\int_{a}^{t} x(s) d s
\end{aligned}
$$

for all $t \in[a, b]$. If there is a constant $K \in(0,1)$ such that the partial derivative $f_{y}$ of $f$ with respect to $y$ exists and

$$
\left|f_{y}(x, y)\right| \leq K
$$

for all $(x, y) \in[a, b] \times \mathbb{R}$. Then, there exists $g \in L_{p}[a, b]$ such that $T g=S g$.

Proof. Let $A=\mathbb{R}^{2}$ with the norm $\left\|\left(u_{1}, u_{2}\right)\right\|=\left|u_{1}\right|+\left|u_{2}\right|$ and $P=\left\{\left(u_{1}, u_{2}\right) \in A\right.$ : $\left.u_{1}, u_{2} \geq 0\right\}$. It is clearly seen that $A$ is a Banach algebra with the multiplication

$$
u v=\left(u_{1}, u_{2}\right)\left(v_{1}, v_{2}\right)=\left(u_{1} v_{1}, u_{1} v_{2}+v_{1} u_{2}\right)
$$


for $u=\left(u_{1}, u_{2}\right), v=\left(v_{1}, v_{2}\right) \in A$ and also $P$ is a cone in $A$. Now, we establish a cone $b$-metric structure $d: X \times X \rightarrow A$ by

$$
d(x, y)=\left(\left\{\int_{a}^{b}|x(t)-y(t)|^{p} d t\right\}^{\frac{1}{p}},\left\{\int_{a}^{b}|x(t)-y(t)|^{p} d t\right\}^{\frac{1}{p}}\right)
$$

for all $x, y \in X$. Using $T$ and $S$ defined above, we obtain from Lagrange mean value theorem

$$
\begin{aligned}
d(\text { Tx, Ty })= & \left(\left\{\int_{a}^{b}\left|\int_{a}^{t} f(s, x(s)) d s-\int_{a}^{t} f(s, y(s)) d s\right|^{p} d t\right\}^{\frac{1}{p}},\right. \\
& \left.\left\{\int_{a}^{b}\left|\int_{a}^{t} f(s, x(s)) d s-\int_{a}^{t} f(s, y(s)) d s\right|^{p} d t\right\}^{\frac{1}{p}}\right) \\
= & \left(\left\{\int_{a}^{b}\left|\int_{a}^{t} f(s, x(s)) d s-f(s, y(s)) d s\right|^{p} d t\right\}^{\frac{1}{p}},\right. \\
& \left.\left\{\int_{a}^{b}\left|\int_{a}^{t} f(s, x(s)) d s-f(s, y(s)) d s\right|^{p} d t\right\}^{\frac{1}{p}}\right) \\
\preceq & \left(K\left\{\int_{a}^{b}\left|\int_{a}^{t} x(s)-y(s) d s\right|^{p} d t\right\}^{\frac{1}{p}}, K\left\{\int_{a}^{b}\left|\int_{a}^{t} x(s)-y(s) d s\right|^{p} d t\right\}^{\frac{1}{p}}\right) \\
= & (K, 0)\left(\left\{\int_{a}^{b}|S x(t)-S y(t)|^{p} d t\right\}^{\frac{1}{p}},\left\{\int_{a}^{b}|S x(t)-S y(t)|^{p} d t\right\}^{\frac{1}{p}}\right) \\
= & (K, 0) d(S x, S y) .
\end{aligned}
$$

Because of

$$
\left\|(K, 0)^{n}\right\|^{\frac{1}{n}}=\left\|\left(K^{n}, 0\right)\right\|^{\frac{1}{n}} \rightarrow K<1(n \rightarrow \infty),
$$

it follows that $\rho((K, 0))<1$. By Corallary 3.6, there exists $g \in L_{p}[a, b]$ such that $T g=$ $S g$.

Note that there may be many concidence points as $g$ for $T$ and $S$, and $T g=S g$ takes the unique value for all $g$. In the sequel, we introduce the condition under which $T$ and $S$ have a unique common fixed point $g^{*} \in L_{p}[a, b]$, that is, $g^{*}$ is the unique solution of system (4.1).

Corollary 4.2. In addition to the conditions given in Theorem 4.1, suppose that the following equality holds:

$$
f\left(s, \int_{a}^{s} g(w) d w\right)=\int_{a}^{s} f(w, g(w)) d w
$$

for all $s \in[a, b]$ and for all $g \in L_{p}[a, b]$ with $S g=T g$. Then, the system (4.1) has a unique solution in $L_{p}[a, b]$.

Proof. By the hypothesis, it is easily seen that $S$ and $T$ are weakly compatible. Thus, all conditions of Corallary 3.6 are satisfied. As a result, $S$ and $T$ have a unique common fixed point $g^{*} \in L_{p}[a, b]$.

As a second application, we now prove the existence and uniqueness of the following coupled equations:

$$
\begin{gathered}
f(x, y)=x \\
g(x, y)=y
\end{gathered}
$$


where $f, g: \mathbb{R}^{2} \rightarrow \mathbb{R}$ are two mappings holding the conditions mentioned below.

Theorem 4.3. If there exist $0<L<1$ and $k>0$ such that

$$
\begin{aligned}
& \left|f\left(x_{1}, y_{1}\right)-f\left(x_{2}, y_{2}\right)\right| \leq L\left|x_{1}-x_{2}\right|, \\
& \left|g\left(x_{1}, y_{1}\right)-g\left(x_{2}, y_{2}\right)\right| \leq L\left|y_{1}-y_{2}\right|+k\left|x_{1}-x_{2}\right|,
\end{aligned}
$$

for all $\left(x_{1}, y_{1}\right),\left(x_{2}, y_{2}\right) \in \mathbb{R}^{2}$. Then there is a unique pair of $(x, y)$ such that it is a solution of the coupled equation (4.2).

Proof. Let $A=\mathbb{R}^{2}$ with the norm $\left\|\left(u_{1}, u_{2}\right)\right\|=\left|u_{1}\right|+\left|u_{2}\right|$ and $P=\left\{\left(u_{1}, u_{2}\right) \in A\right.$ : $\left.u_{1}, u_{2} \geq 0\right\}$. It is clear that $A$ is a Banach algebra with the multiplication

$$
u v=\left(u_{1}, u_{2}\right)\left(v_{1}, v_{2}\right)=\left(u_{1} v_{1}, u_{1} v_{2}+v_{1} u_{2}\right)
$$

for $u=\left(u_{1}, u_{2}\right), v=\left(v_{1}, v_{2}\right) \in A$ and also $P$ is a cone in $A$. Now, we define a mapping $d: X \times X \rightarrow A$ by

$$
d\left(\left(x_{1}, y_{1}\right),\left(x_{2}, y_{2}\right)\right)=\left(\left|x_{1}-x_{2}\right|,\left|y_{1}-y_{2}\right|\right) .
$$

It is easy to see that $(X, d)$ is a complete cone $b$-metric space over Banach algebra $A$ with the coefficient $s=1$. Using mappings $T$ and $S$ defined as follows:

$$
S(x, y)=(x, y), T(x, y)=(f(x, y), g(x, y)),
$$

we have

$$
\begin{aligned}
d\left(T\left(x_{1}, y_{1}\right), T\left(x_{2}, y_{2}\right)\right) & =d\left(\left(f\left(x_{1}, y_{1}\right), g\left(x_{1}, y_{1}\right)\right),\left(f\left(x_{2}, y_{2}\right), g\left(x_{2}, y_{2}\right)\right)\right) \\
& =\left(\left|f\left(x_{1}, y_{1}\right)-f\left(x_{2}, y_{2}\right)\right|,\left|g\left(x_{1}, y_{1}\right)-g\left(x_{2}, y_{2}\right)\right|\right) \\
& \preceq\left(L\left|x_{1}-x_{2}\right|, L\left|y_{1}-y_{2}\right|+k\left|x_{1}-x_{2}\right|\right) \\
& =(L, k)\left(\left|x_{1}-x_{2}\right|,\left|y_{1}-y_{2}\right|\right) \\
& =(L, k) d\left(S\left(x_{1}, y_{1}\right), S\left(x_{2}, y_{2}\right)\right) .
\end{aligned}
$$

Since

$$
\left\|(L, k)^{n}\right\|^{\frac{1}{n}}=\left\|\left(L^{n}, k n L^{n-1}\right)\right\|^{\frac{1}{n}}=\left(L^{n}+k n L^{n-1}\right)^{\frac{1}{n}} \rightarrow L<1(n \rightarrow \infty),
$$

it follows that $\rho((L, k))<1$. By Corallary 3.6, there exists a unique pair of $\left(x^{*}, y^{*}\right) \in \mathbb{R}^{2}$ such that satisfies the coupled equation (4.2).

Acknowledgment. The authors thank the referee for some useful comments and suggestions.

\section{References}

[1] M. Abbas and G. Jungck, Common fixed point results for noncommuting mappings without continuity in cone metric spaces, J. Math. Anal. Appl. 341 (1), 416-420, 2008.

[2] V. Berinde, Approximating fixed points of weak contractions using the Picard iteration, Nonlinear Anal. Forum, 9 (1), 43-53, 2004.

[3] W.S. Du, A note on cone metric fixed theory and its equivalence, Nonlinear Anal. 72 (5), 2259-2261, 2010.

[4] L.G. Huang and X. Zhang, Cone metric spaces and fixed point theorems of contractive mappings, J. Math. Anal. Appl. 332 (2) 1468-1476, 2007.

[5] H. Huang and S. Radenovic, Common fixed point theorems of generalized Lipschitz mappings in cone b-metric spaces over Banach algebras and applications, J. Non. Sci. Appl. 8 (5) , 787-799, 2015.

[6] H. Huang, S. Hu, B.Z. Popovic and S. Radenovic, Common fixed point theorems for four mappings on cone b-metric spaces over Banach algebras, J. Non. Sci. Appl. 9 (6), 3655-3671, 2016. 
[7] G. Jungck, Commuting mappings and fixed points, Amer. Math. Monthly, 83 (4), 261-263, 1976.

[8] G. Jungck, Common fixed points for noncontinuous nonself maps on non-metric spaces, Far East J. Math. Sci. 4 (2), 199-215, 1996.

[9] Z. Kadelburg and S. Radenovic, A note on various types of cones and fixed point results in cone metric spaces, Asian J. Math. Appl. 2013, Article ID:ama0104, 2013.

[10] H. Liu and S. Xu, Cone metric spaces with Banach algebras and Fixed point theorems of generalized Lipschitz mappings, Fixed Point Theory Appl. 2013 (320), 2013.

[11] M. Ozavsar, Fixed point theorems for $(k, l)$-almost contractions in cone metric spaces over Banach algebras, Mathematical Advances in Pure and Applied Sciences, 1 (1), 46-51, 2018.

[12] Y. Piao, Unique common fixed points for two mappings with Kannan-Chatterjea type conditions on cone metric spaces over Banach algebras without normality, Adv. Inequal. Appl. 2016, Article ID: 1, 2016.

[13] S. Radenovic and B.E. Rhoades, Fixed point theorem for two non-self mappings in cone metric spaces, Comput. Math. Appl. 57 (10), 1701-1707, 2009.

[14] W. Rudin, Functional Analysis and its Applications, McGraw-Hill, New York, 1991.

[15] T. Suzuki, Basic inequality on a b-metric space and its applications, J. Inequal. Appl. 2017, 256, 2017.

[16] S. Xu and S. Radenovic, Fixed point theorems of generalized Lipschitz mappings on cone metric spaces over Banach algebras without assumption of normality, Fixed Point Theory Appl. 2014, 102, 2014. 